\title{
From brane dynamics to a Kac-Moody invariant formulation of $\mathrm{M}$-theories
}

\author{
François Englert ${ }^{* a}$ and Laurent Houart ${ }^{\dagger b}$ \\ ${ }^{a}$ Service de Physique Théorique \\ Université Libre de Bruxelles, Campus Plaine, C.P.225 \\ Boulevard du Triomphe, B-1050 Bruxelles, Belgium \\ ${ }^{b}$ Service de Physique Théorique et Mathématique and International Solvay Institutes \\ Université Libre de Bruxelles, Campus Plaine C.P. 231 \\ Boulevard du Triomphe, B-1050 Bruxelles, Belgium \\ E-mail: 'fenglert@ulb.ac.be lhouart@ulb.ac.bé
}

ABSTRACT: Theories of gravity coupled to forms and dilatons may admit as solutions zero binding energy configurations of intersecting closed extremal branes. In such configurations, some branes may open on host closed branes. Properties of extremal branes reveal symmetries of the underlying theory which are well known in M-theory but transcend supersymmetry. From these properties it is possible to reconstruct all actions, comprising in particular pure gravity in $D$ dimensions, the bosonic effective actions of M-theory and of the bosonic string, which upon dimensional reduction to three dimensions are invariant under the maximally non-compact simple simply laced Lie groups $\mathcal{G}$. Moreover the features of extremal branes suggest the existence of a much larger symmetry, namely the 'very-extended' Kac-Moody algebras $\mathcal{G}^{+++}$. This motivates the construction of explicit non-linear realisations of all simple $\mathcal{G}^{+++}$, which hopefully contain new degrees of freedom such as those encountered in string theories. They are defined without a priori reference to space-time and are proposed as substitutes for original field theoretic models of gravity, forms and dilatons. From the $\mathcal{G}^{+++}$-invariant theories, all algebraic properties of extremal branes are recovered from exact solutions, and there are indications that space-time is hidden in the infinite symmetry structure. The transformation properties of the exact solutions, which possibly induce new solutions foreign to conventional theories, put into evidence the general group-theoretical origin of 'dualities' for all $\mathcal{G}^{+++}$. These dualities apparently do not require an underlying string theory.

\footnotetext{
${ }^{*}$ Speaker.

${ }^{\dagger}$ Research Associate F.N.R.S.
} 


\section{Introduction and conclusion}

Theories of gravity coupled to forms and dilatons may admit as solutions zero binding energy configurations of intersecting closed extremal branes [i⿱亠䒑口] $]$. In such configurations, some branes may open on host closed branes [2] symmetries of the underlying theory, which are well known in M-theory but transcend supersymmetry. From these properties it is possible to reconstruct all actions, comprising in particular pure gravity in $D$ dimensions, the bosonic effective actions of M-theory and of the bosonic string, which upon dimensional reduction to three dimensions are invariant

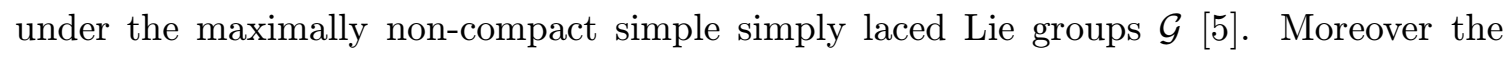
features of extremal branes suggest the existence of a much larger symmetry, namely the 'very-extended' Kac-Moody algebras $\mathcal{G}^{+++}$. Such symmetries were first conjectured in

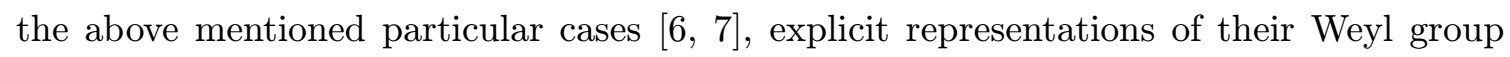
for Kasner-type solutions were obtained for all simple $\mathcal{G}^{+++}$[8]

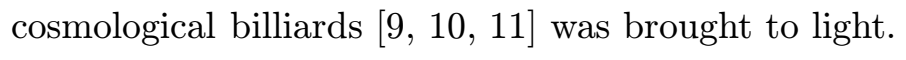

These facts motivate the construction of explicit non-linear realisations of all simple $\mathcal{G}^{+++}$on cosets obtained from a modified Chevalley involution [i] $\left.{ }_{-1}^{2}\right]$. The $\mathcal{G}^{+++}$-invariant actions are proposed as substitutes for the original field theoretic models of gravity, forms and dilatons, and hopefully contain new degrees of freedom such as those encountered in string theories. The actions are defined on a world-line a priori unrelated to spacetime. The latter should then be deduced dynamically. Such an approach to gravity and forms, if successful, would dispose of the need of explicit diffeomorphism invariance or gauge invariance. All such information should be hidden in the global $\mathcal{G}^{+++}$invariance. Although it may seem that global symmetries cannot contain local symmetries, in particular in view of the celebrated Elitzur theorem [1] ${ }_{1}^{1} \overline{3}$, it need not be the case in view of the infinite number of generators of $\mathcal{G}^{+++}$. We formulate the $\mathcal{G}^{+++}$invariant theory recursively from a level

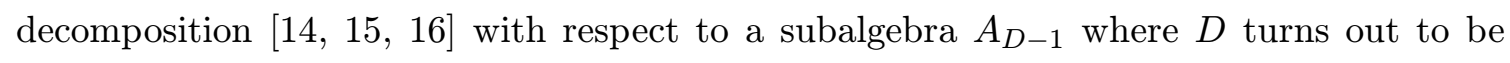
the space-time dimension. Our formulation is exploratory and does not pretend to be a final one. No attempt is made to cope with fermionic degrees of freedom and we limit here our investigation to the classical domain. From the $\mathcal{G}^{+++}$-invariant theories, all algebraic properties of extremal branes are recovered from exact solutions, and there are indications that space-time is hidden in the infinite level structure. The transformation properties of the exact solutions, which also generate Kaluza-Klein waves and KK-monopoles (TaubNUT spaces) and possibly solutions foreign to conventional theories, put into evidence the general group-theoretical origin of 'dualities' for all $\mathcal{G}^{+++}$. These dualities apparently do not require an underlying string theory.

In Sections 2 and 3, we review the properties of intersecting extremal branes and of their opening, which are used in Section 4 to reconstruct dynamically the simple simply laced Lie algebras $\mathcal{G}$ and provide motivation for considering the very-extended Kac-Moody algebras $\mathcal{G}^{+++}$. The non-linear realisations of $\mathcal{G}^{+++}$are presented in Section 5. Extremal branes and their duality properties are revisited in this new framework in Section 6 . 


\section{Intersection rules for extremal branes}

We begin with a generic theory in $D$ dimensions which includes gravity, one dilaton and $\mathcal{M}$ field strengths of arbitrary form degree $p_{I}$ with $p_{I} \leq D / 2$ and arbitrary couplings to the dilaton $a_{I}$. The action is

$$
S=\frac{1}{16 \pi G_{N}^{(D)}} \int d^{D} x \sqrt{-g}\left(R-\frac{1}{2}(\partial \phi)^{2}-\sum_{I} \frac{1}{2 p_{I} !} e^{a_{I} \phi} F_{p_{I}}^{2}\right), \quad I=1 \ldots \mathcal{M},
$$

where we have not included possible Chern-Simons terms. It can be shown that they have no effect on the zero binding energy solutions considered here although such terms will be important in the following sections.

The 'BPS' zero binding energy configurations of closed extremal branes intersecting orthogonally are obtained by first specialising to metrics of the following diagonal form,

$$
d s^{2}=-e^{2 p^{(1)}} d \tau^{2}+\sum_{\mu=2}^{D-p} e^{2 p^{(\mu)}}\left(d x^{\mu}\right)^{2}+\sum_{\lambda=1}^{p} e^{2 p^{(D-p+\lambda)}}\left(d y^{\lambda}\right)^{2},
$$

where $y^{\lambda}$ label $p$ compact coordinates. The functions $p^{(\alpha)}(\alpha=1,2, \ldots D)$ depend only on the transverse coordinates $x^{\mu}$ in the non-compact dimensions and allow for multi-centre solutions. We choose $p$ so that all branes are wrapped in the compact dimensions and that no compact dimension is transverse to all branes. Thus for each brane $p \geq q_{A}$ where $q_{A}$ is the dimension of the brane. If $q_{A}<p$, we take a lattice of $q_{A}$-branes in the compact directions transverse to the brane and average over them. Here $q_{A}$ designates either an electrically charged $q_{A}^{e}$-brane with respect to a $p_{I}$-form field strength $F_{p_{I}}$, or its dual $q_{A}^{m}$ magnetic brane. For $\mathcal{N}$ intersecting branes, we write

$$
p^{(\alpha)}=\sum_{A=1}^{\mathcal{N}} p_{A}^{(\alpha)} \quad \text { and } \quad \phi=\sum_{A=1}^{\mathcal{N}} \phi_{A}
$$

with

$$
(p+3-D) p_{A}^{(\mu)}=p_{A}^{(1)}+\sum_{\lambda=1}^{p} p_{A}^{(D-p+\lambda)} \quad \mu=2, \ldots, D-p .
$$

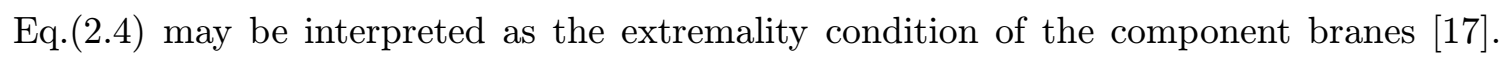
For electric and magnetic brane potentials, we take

$$
\begin{aligned}
& \text { Electric : } A_{\tau \lambda_{1} \ldots \lambda_{q_{A}^{e}}}=\epsilon_{\tau \lambda_{1} \ldots \lambda_{q_{A}}} E_{A}^{e}\left(\left\{x^{\nu}\right\}\right), \\
& \text { Magnetic }: \widetilde{A}_{\tau \lambda_{1} \ldots \lambda_{q_{A}^{m}}}=\epsilon_{\tau \lambda_{1} \ldots \lambda_{q_{A}^{m}}} E_{A}^{m}\left(\left\{x^{\nu}\right\}\right),
\end{aligned}
$$

where $\widetilde{A}$ is the (magnetic) potential of the dual field strength $\widetilde{F}$ defined by

$$
\sqrt{-g} e^{a_{I} \phi} F^{\mu_{1} \ldots \mu_{p_{I}}}=\frac{1}{\left(D-p_{I}\right) !} \epsilon^{\mu_{1} \ldots \mu_{p_{I}} \nu_{1} \ldots \nu_{D-p_{I}}} \widetilde{F}_{\nu_{1} \ldots \nu_{D-p_{I}}} .
$$


Using these ansätze, the Einstein equations and the equations for the dilaton and the forms yield

$$
\begin{aligned}
p_{A}^{(1)} & =-\frac{D-q_{A}-3}{\Delta} \ln H_{A}, \\
p_{A}^{(\mu)} & =\frac{q_{A}+1}{\Delta} \ln H_{A}, \\
p_{A}^{(D-p+\lambda)} & =\frac{\delta_{A}^{(\lambda)}}{\Delta} \ln H_{A}, \\
\phi_{A} & =\frac{D-2}{\Delta} \varepsilon_{A} a \ln H_{A},
\end{aligned}
$$

where $H_{A}\left(\left\{x^{\nu}\right\}\right)$ is a harmonic function related to the $E_{A}$ by $H_{A}=\sqrt{2(D-2) / \Delta} E_{A}^{-1}$ and

$$
\Delta=\left(q_{A}+1\right)\left(D-q_{A}-3\right)+\frac{1}{2} a_{A}^{2}(D-2) .
$$

In Eq. $(2 . \overline{2} \cdot \overline{8}) \delta_{A}^{(\lambda)}=-\left(D-q_{A}-3\right)$ or $\left(q_{A}+1\right)$ depending on wether $y^{\lambda}$ is parallel or perpendicular to the $q_{A}$-brane. The factor $\varepsilon_{A}$ is +1 for an electric brane and -1 for a magnetic one. The harmonic functions $H_{A}\left(\left\{x^{\nu}\right\}\right)$ allow for parallel branes and are given (for $D-p>3$ ) by

$$
H_{A}=1+\sum_{k} \frac{Q_{k}}{\left|x^{\mu}-x_{k}^{\mu}\right|^{D-p-3}},
$$

where the $x_{k}^{\mu}$ label the positions in non-compact space-time of the branes with charge $Q_{k}$. For $\mathcal{N}>1$, Eqs. $(2.8)$ are restricted by algebraic conditions stemming from the equation for the non diagonal components of the curvature tensor. These are, for each pair $(A, B)$ of distinct $q$-branes of dimensions $\left(q_{A}, q_{B}\right)$, the number of dimensions $\bar{q}\left(-1 \leq \bar{q} \leq q_{A}, q_{B}\right)$ on which they intersect ${ }^{0}$ in terms of the total number of space-time dimensions $D$ and of the field strength couplings to the dilaton. These intersection rules read [i] $\left.\bar{i}_{1}\right]$

$$
\bar{q}+1=\frac{\left(q_{A}+1\right)\left(q_{B}+1\right)}{D-2}-\frac{1}{2} \varepsilon_{A} a_{A} \varepsilon_{B} a_{B} .
$$

Note that such zero energy binding configurations were originally considered in the context of M-theory 'phases' but may arise classically without supersymmetry.

\section{The opening of branes}

We now analyse the breaking of closed extremal branes into open branes terminating on closed ones. We consider the above BPS configurations in the special case when $\bar{q}$ has the same dimension as the potential boundary of one of the two constituent branes, i.e $q_{A}-1$ or $q_{B}-1$, and study its possible opening. Such opening requires the addition of ChernSimons terms to the action Eq.(- $\left(\begin{array}{l}1 \\ 2\end{array}\right)$ and may enlarge the brane content of the theory. We shall see in Section 4 that, under some conditions, such openings fully determine the theory

\footnotetext{
${ }^{0} \bar{q}$ must be integer and the case $\bar{q}=-1$ is relevant. It can be interpreted in terms of instantons in the Euclidean, in which case the time coordinate need not be longitudinal to all branes.
} 
and relate brane dynamics to the existence of a symmetry. The presentation given in this section is a generalisation of the one performed in the context of M-theory [2,

Let us review how extended objects carrying a conserved charge can be opened. The main obstacle towards opening of branes is charge conservation. Generically, the charge density of a $q$-brane is measured by performing an integral of the relevant field strength on a $(D-q-2)$-dimensional sphere $S^{D-q-2}$ surrounding the brane in its transverse space,

$$
Q_{q} \propto \int_{S^{D-q-2}} * F_{q+2} .
$$

If the brane is open, we can slide the $S^{D-q-2}$ off a loose end and shrink it to zero size. This would imply the vanishing of the charge and hence a violation of charge conservation. This conclusion is avoided if, in the above process, the $S^{D-q-2}$ necessarily goes through a region in which the equation

$$
d * F_{q+2}=0
$$

no longer holds. This is the case when the open brane ends on some other one.

In the framework of M-theory the source terms needed in Eq. $(\overline{3} \cdot \overline{2} \cdot \bar{i})$ to ensure charge conservation for the open branes originate from two requirements whose interplay leads to a consistent picture. On the one hand there are space-time Chern-Simons type terms in supergravity which allow for charge conservation for well defined pairing of open and 'host' branes [3in]. On the other hand the world-volume effective actions [2] for the branes of Mtheory relate world-volume fields and pullbacks of space-time fields, and gauge invariance [i] $1 \overline{1} \overline{8}]$ for open branes ending on the 'host' brane implies that the end of the open branes acts as a source for the world-volume field living on the closed 'host' brane.

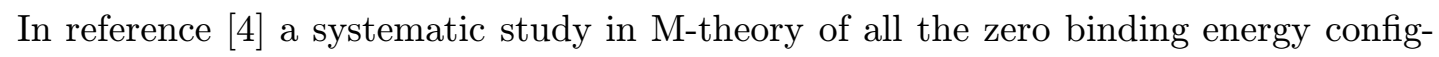
urations Eq. $(2 . \overline{1} \overline{1})$ ) corresponding to $\bar{q}=q_{A}-1$ (with $q_{A} \leq q_{B}$ ) was performed. It was shown that in all cases it was possible to open the $q_{A}$-brane along its intersection with the $q_{B}$-brane. The crucial ingredient was the presence of the appropriate Chern-Simons terms in the supergravity Lagrangians for each case.

Here we propose to reverse the logic. Starting with a Lagrangian of type Eq. $\left(\overline{2} \cdot \overline{1} \cdot \frac{1}{1}\right)$ and having zero binding energy configurations between branes we will ask that, if $\bar{q}=q_{A}-1$, the corresponding $q_{A}$-brane open on the $q_{B}$-brane. This will determine the form of the

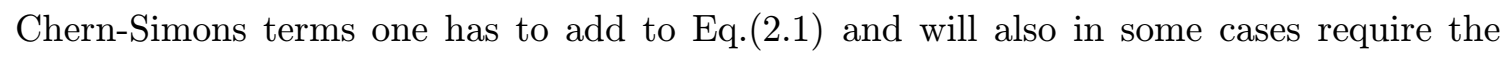
introduction of new field strength forms $F_{n_{I}}$. One then proceeds iteratively.

We illustrate the role of the Chern-Simons term (see also a theory with only one $n$-form $F_{q^{e}+2}$ and dilaton coupling in Eq.( $\left(\overline{2} .1_{1}^{\prime}\right)$ such that the intersection rule between the electric $q^{e}$-brane and the magnetic $q^{m}$-brane $\left(q^{m}=D-q^{e}-4\right)$ is $\bar{q}=q^{e}-1$.

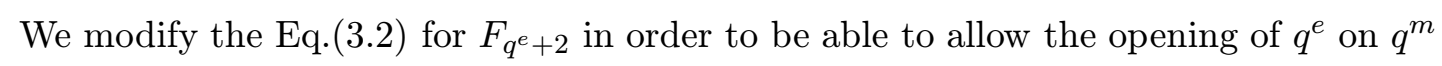
by the addition to the action Eq. $(2 \cdot 1) \cdot 1)$ of the Chern-Simons term

$$
\int A_{q^{e}+1} \wedge F_{q^{e}+2} \wedge F_{D-2 q^{e}-3}
$$


and of a standard kinetic energy term for the new ${ }^{1}$ field strength $F_{D-2 q^{e}-3}$. Its dilaton coupling is chosen such that the intersection rules Eq.(2.1.1in) give integer intersection dimension between the new extremal electric $\left(D-2 q^{e}-5\right)$-brane and its dual magnetic $\left(2 q^{e}+1\right)$-brane. Charge conservation now reads

$$
d * F_{q^{e}+2}=F_{q^{e}+2} \wedge F_{D-2 q^{e}-3}+Q^{e} \delta_{D-q^{e}-1} .
$$

Here wedge products are defined up to signs and numerical factors. In the r.h.s. of Eq.(3.4) the first term comes from the variation of the Chern-Simons term and the second one is the $q^{e}$-brane charge density. $\delta_{D-q^{e}-1}$ is the Dirac delta function in the directions transverse to the $q^{e}$-brane. We introduce here an explicit source term for the electric brane since, to study its opening, we want to extend to the branes themselves the validity of the usual closed brane solution. Such term is required because the equations of motion from which the intersecting brane solutions were derived do not contain any source term and are therefore valid only outside the sources.

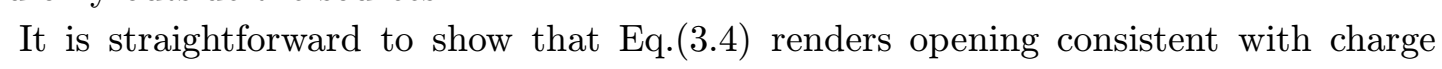
conservation and that new branes arising from $F_{D-2 q^{e}-3}$ have to be added. From Eqs.(3.3. and $\left(\overline{3} . \overline{4}^{\prime}\right)$, the addition of such branes can be determined in a picturesque way. Namely, when a brane opens on a closed 'host' brane, the boundary appears from the world-volume point of view as a charged object under a world-volume field strength living in the closed brane. The world-volume Hodge dual of this object is the boundary of an other brane which can also be consistently opened on the same closed 'host' brane. The field strength associated to this new brane is precisely the one appearing in the Chern-Simons ensuring the consistency of the opening of the brane we started with.

In brief, having a theory of type Eq. $(\overline{2} \cdot \overline{2} \cdot \overline{1})$ in which some zero binding energy configurations give potential boundaries, it is possible to complete it in a well-defined way by adding Chern-Simons terms ${ }^{2}$, and when necessary new form field strengths (hence new branes) in order to ensure consistency of brane opening with charge conservation. In section 4 , such dynamical requirement will be at work to reconstruct purely from brane considerations theories whose dimensional reduction is characterised by a coset symmetry.

\section{From brane dynamics to $\mathcal{G}$ and $\mathcal{G}^{+++}$}

\subsection{Intersection rules and dimensional reduction}

We perform the dimensional reduction to three dimensions of a generic theory described by an action Eq.( $\left(\overline{2}_{2}^{2} \cdot \overline{1}_{1}\right)$, possibly with the addition of Chern-Simons terms and discuss the possible emergence of a coset symmetry. We relate the onset of such symmetry to the intersection rules for BPS configurations between the extremal branes of the theory considered.

\footnotetext{
${ }^{1}$ If $D=3 q^{e}+5$ there is no need to introduce a new field strength as the Chern-Simons terms can be build with the field strength $F_{q^{e}+2}$.

${ }^{2}$ The coefficients of the Chern-Simons terms Eq. $\left({ }_{3}^{-} \cdot 3^{i}\right)$ are not fixed in this qualitative discussion. Their precise values are important when one will discuss the potential symmetries. In the framework of M-theory they are usually fixed by supersymmetry. Nevertheless it is possible to fix them or at least quantise them

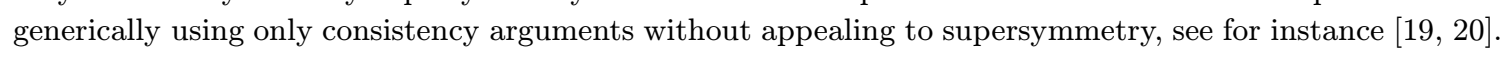


Starting with the theory defined in $D$ dimensions, we compactify to $D-1$ dimensions while remaining in the Einstein frame with the standard 1/2 kinetic term normalisation for the new scalar. We use the notation $\phi_{2}$ for the scalar appearing in the first step of the dimensional reduction and rename $\phi_{1}$ the dilaton $\phi$ already present in the uncompactified theory defined by Eq.(2). $\left.\overline{2}_{1}^{1}\right)$. The compactified coordinate is $x^{D-1}$, the uncompactified coordinates are $x^{\mu}$ where $\mu=0 \ldots D-2$, and

$$
\beta_{D}=\sqrt{\frac{1}{2(D-1)(D-2)}} .
$$

The gravitation part of the action Eq.(20.1) becomes

$$
\begin{aligned}
\int d^{D} x \sqrt{-g_{D}} R_{D} & =\int d^{D-1} x \sqrt{-g_{D-1}}\left(R_{D-1}-\frac{1}{2} \partial_{\mu} \phi_{2} \partial^{\mu} \phi_{2}\right. \\
& \left.-\frac{1}{4} e^{-2(D-2) \beta_{D-1} \phi_{2}} F_{\mu \nu} F^{\mu \nu}\right) .
\end{aligned}
$$

where $F_{\mu \nu}=\partial_{\mu} A_{\nu}-\partial_{\nu} A_{\mu}$.

For each $n_{I}$-form field strength $F_{n_{I}}$ in Eq.( $\left(\begin{array}{l}2 \\ 2\end{array} . \overline{1} i_{1}^{1}\right)$ we get after reduction

$$
\begin{aligned}
\int d^{D} x \sqrt{-g_{D}} \frac{1}{2 n_{I} !} e^{a_{I} \phi_{1}} F_{n_{I}}^{2} & =\int d^{D-1} x \sqrt{-g_{D-1}}\left(\frac{1}{2 n_{I} !} e^{a_{I} \phi_{1}-2\left(n_{I}-1\right) \beta_{D-1} \phi_{2}} F_{n_{I}}^{2}\right. \\
& \left.+\frac{1}{2\left(n_{I}-1\right) !} e^{a_{I} \phi_{1}+2\left(D-1-n_{I}\right) \beta_{D-1} \phi_{2}} F_{n_{I}-1}^{2}\right),
\end{aligned}
$$

where

$$
F_{\mu_{1} \ldots \mu_{n}}^{\prime}=F_{\mu_{1} \ldots \mu_{n}}-n F_{\left[\mu_{1} \ldots \mu_{n-1}\right.} A_{\left.\mu_{n}\right]} .
$$

We can repeat this procedure step by step to obtain the theory on a $p$-torus. One has then obviously $p$ scalars $\phi_{j}$ with $j=2 \ldots p+1$ parametrising the radii of the torus, coming from the diagonal components of the metric in the compact dimensions. Additional scalars denoted $\chi_{\vec{\alpha}}$ arise from several origins. They come from potentials $A_{\mu}^{k}$ which arise when reducing gravity from $D+1-k$ to $D-k$ and also from the potentials associated to the $F_{n_{I}}$ (when $p \geq n_{I}-1$ ) with indices in the compact dimensions. In addition the $n$-form field strengths give additional scalars when $p=D-n-1$ by dualising them. In particular when we reach $D=3$ the $F_{\mu \nu}^{k}$ (with $k=1 \ldots D-3$ ) coming from the gravity part of the action (i.e. the graviphotons) can be dualised to scalars, and we are left with only scalars. The action takes then the form

$$
S=\int d^{3} x \sqrt{-g_{3}}\left(R_{3}-\frac{1}{2} \partial_{\mu} \vec{\phi} \cdot \partial^{\mu} \vec{\phi}-\frac{1}{2} \sum_{\vec{\alpha}} e^{\sqrt{2} \vec{\alpha} \cdot \vec{\phi}} \partial_{\mu} \chi_{\vec{\alpha}} \partial^{\mu} \chi_{\vec{\alpha}}+\ldots\right)
$$

where $\vec{\phi}=\left(\phi_{D-2}, \ldots, \phi_{1}\right)$, the $\vec{\alpha}$ are constant $(D-2)$-vectors ${ }^{3}$ characterising each $\chi_{\vec{\alpha}}$. If we start in $D$ dimensions without dilaton the vectors are of course $(D-3)$-dimensional as $\phi_{1}$ is absent in that case. The ellipsis in Eq.(14.5ind stands for terms of order higher

\footnotetext{
${ }^{3}$ The normalisation factor $\sqrt{2}$ has been chosen for convenience. It will eventually correspond in the simply laced case to the standard normalisation of the roots, namely $\vec{\alpha} \cdot \vec{\alpha}=2$.
} 
than quadratic in the $\chi_{\vec{\alpha}}$ scalars. They come from the modification of the field strengths Eq.(4.4. in the uncompactified theory.

The action Eq. $(\overline{2} \cdot \overline{1} \cdot \overline{1})$ dimensionally reduced to three dimensions has a $\mathcal{G} / \mathcal{H}$ symmetry if the vectors $\vec{\alpha}$ obtained from the compactification can be identified with the positive roots of a group $\mathcal{G}$ and if, when necessary, some precise Chern-Simons terms are added in the uncompactified theory $[2 \overline{1} \overline{1}]$. The requirement that the $\vec{\alpha}$ correspond to positive roots is thus a necessary condition to uncover a symmetry [i].

Recall first the well-known dimensional reduction of pure gravity (see Eq.(4.42.24)) down to three dimensions, which leads to $\mathcal{G}=S L(D-2)$ whose algebra is $A_{D-3}$. The scalars corresponding to the simple roots of $A_{D-3}$ are of two kinds.

There are first $D-4$ scalars which are the components $A_{D-k-1}^{k}$ of the potentials coming from $g_{D-k, D-k-1}, k=1 \ldots D-4$. These are obtained by performing the "fastest"

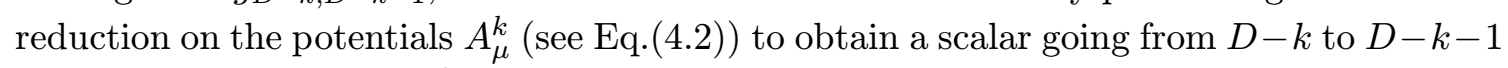
when compactifying on $T^{k+1}$. The corresponding simple roots $\vec{\alpha}_{k}^{g}$ are given by

$$
\begin{aligned}
\vec{\alpha}_{k}^{g} & =\sqrt{2}(\underbrace{0, \ldots, 0}_{D-4-k \text { terms }},(D-k-3) \beta_{D-k-1},-(D-k-1) \beta_{D-k}, \underbrace{0, \ldots, 0}_{k-1 \text { terms }} ; 0), \\
k & =1 \ldots D-4 .
\end{aligned}
$$

They define a subalgebra $A_{D-4}$. We have indeed

$$
\vec{\alpha}_{k}^{g} \cdot \vec{\alpha}_{l}^{g}=\left\{\begin{array}{cc}
2 & k=l \\
-1 & |k-l|=1 \\
0 & |k-l| \geq 2
\end{array}\right.
$$

Reading from right to left, the first component of $\vec{\alpha}_{k}^{g}$ associated to the dilaton $\phi$ in the

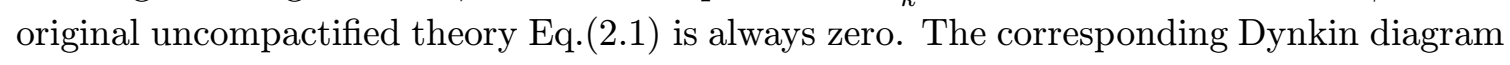
with $D-4$ nodes, which from now on we will refer to as the gravity line, is depicted in Fig.1.

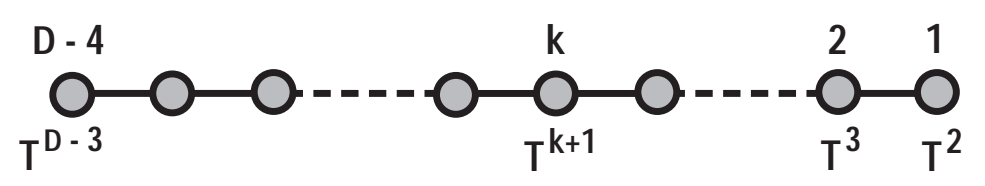

Fig.1. The gravity line.

Dynkin diagram of $A_{D-4}$ generated by the dimensional reduction to 3 dimensions.

The remaining scalar corresponding to the missing simple root leading to the full $A_{D-3}$ comes from dualising in three dimensions the first vector (graviphoton) that arises in the stepwise procedure namely the vector appearing already in $D-1$ dimensions. The corresponding simple root is

$$
\vec{\alpha}^{g p}=\sqrt{2}\left(\beta_{3}, \ldots, \beta_{D-3}, \beta_{D-2},(D-2) \beta_{D-1} ; 0\right) .
$$


Note that this simple root $\vec{\alpha}^{g p}$ has a non-vanishing scalar product with $\vec{\alpha}_{1}^{g}$ (i.e with the simple root already appearing when compactifying on $T^{2}$ ). One has indeed $\vec{\alpha}_{k}^{g} \cdot \vec{\alpha}^{g p}=-\delta_{k, 1}$. Consequently it attaches itself to the right of the gravity line. The other $\frac{1}{2}(D-4)(D-3)$ scalars coming from the reduction of gravity down to three dimensions give all the positive roots of $A_{D-3}$.

We now turn to theories with forms given by Eq.( $\left(\overline{2} . \overline{1}_{1}^{1}\right)$ and consider a $n_{A}$-form $F_{n_{A}}$ with dilaton coupling $a_{A}$ (and $n_{A} \leq D / 2$ ). Let us consider the first scalar arising from the $n_{A}$-form upon dimensional reduction up to $p=n_{A}-1$. The vector $\vec{\alpha}_{n_{A}}^{e}$ associated to this scalar $^{4}$ will from now on be called the would-be electric root. It is given by

$$
\vec{\alpha}_{n_{A}}^{e}=(\underbrace{0, \ldots, 0}_{D-n_{A}-2 \text { terms }}, \underbrace{b_{\left(n_{A}, D\right)} \beta_{D-n_{A}+1}, \ldots, b_{\left(n_{A}, D\right)} \beta_{D-2}, b_{\left(n_{A}, D\right)} \beta_{D-1}}_{n_{A}-1 \text { terms }} ; \frac{a_{A}}{\sqrt{2}}),
$$

with

$$
b_{\left(n_{A}, D\right)}=\sqrt{2}\left(D-n_{A}-1\right) .
$$

First, we compute the scalar product of the would-be electric root with the gravity line ${ }^{5}$.

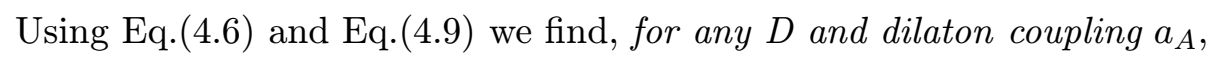

$$
\vec{\alpha}_{k}^{g} \cdot \vec{\alpha}_{n_{A}}^{e}=-\delta_{k, n_{A}-1}
$$

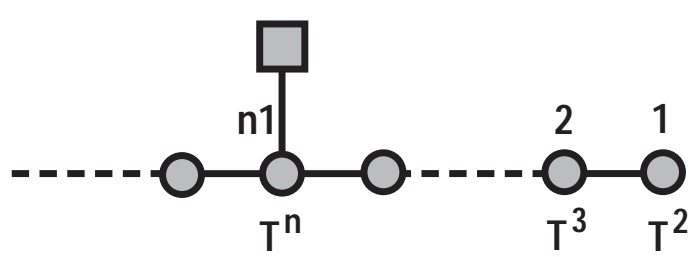

Fig.2. Would-be Dynkin diagram.

The would-be electric root associated to $F_{n}$ is represented by a shaded square.

Using the scalar product Eq.(4.11) of the would-be electric root with the gravity line, we can draw a would-be Dynkin diagram where the would-be electric root associated to $F_{n_{A}}$ is connected to the $\left(n_{A}-1\right)^{t h}$ node of the gravity line as depicted in Fig.2.

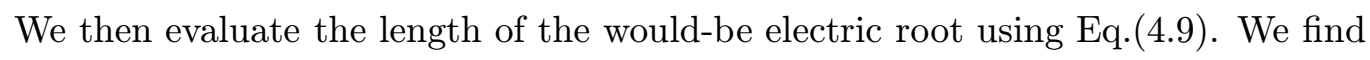

$$
\begin{aligned}
\vec{\alpha}_{n_{A}}^{e} \cdot \vec{\alpha}_{n_{A}}^{e} & =\frac{\left(n_{A}-1\right)\left(D-n_{A}-1\right)}{(D-2)}+\frac{a_{A}^{2}}{2} \\
& =\frac{\left(q_{A}^{e}+1\right)\left(q_{A}^{m}+1\right)}{(D-2)}+\frac{a_{A}^{2}}{2} \\
& =\bar{q}^{\left(e_{A}, m_{A}\right)}+1 .
\end{aligned}
$$

\footnotetext{
${ }^{4}$ The other scalars obtained by further dimensional reduction give $\vec{\alpha}$-vectors that are linear combinations with positive integer coefficients of $\vec{\alpha}_{n_{A}}^{e}$ and of the $\vec{\alpha}_{k}^{g}$.

${ }^{5}$ The scalar product of the would-be electric root with the graviphoton is one for any $D$ and $a_{A}$. This implies that when forms are present in a theory with symmetry, the graviphoton is never a simple root. Consequently we focus on the gravity line.
} 
We thus see that the square length of the would-be electric root associated to $F_{n_{A}}$ can be written in terms of the intersection rule equation Eq.(2.11) giving the intersection between the electric and the magnetic brane charged under $F_{n_{A}}$.

From now on we will restrict ourselves to simply laced groups. In our normalisation all their roots are of square length two. In order for the would-be root to be a root, one must have $\vec{\alpha}_{n_{A}}^{e} \cdot \vec{\alpha}_{n_{A}}^{e}=2$. Consequently the existence of a BPS configuration in the original theory, consisting of an electric extremal p-brane $(p \geq 1)$ and its magnetic dual whose intersection is $\bar{q}^{\left(e_{A}, m_{A}\right)}=1$, is a necessary condition to have after dimensional reduction an enhanced simply laced Lie group symmetry.

\subsection{Dynamical reconstruction of Lie groups}

Our starting point is a theory given by Eq. $\left(\overline{2}_{2} .1_{1}^{3}\right)$ in $D$ dimensions with only one $n_{A}$-form field strength $F_{n_{A}}$ and its dilaton coupling $a_{A}$. We will fix the dilaton coupling such that there exists a zero-binding energy configuration between the electric $q_{A}^{e}$-brane $\left(q_{A}^{e}=n_{A}-2\right)$ and the magnetic $q_{A}^{m}$-brane with $\bar{q}^{\left(e_{A}, m_{A}\right)}=1$. As explained in the previous section this is a necessary condition in order to find a new symmetry. Once the dilaton coupling of the form is fixed, we require that, when the dimensionality of an intersection permits opening, the latter is consistent with charge conservation. Namely we impose that, if $\bar{q}^{\left(e_{A}, m_{A}\right)}=q_{A}^{e}-1$, the electric brane open on the magnetic brane. As explained in Section 3, this requires the introduction of a specific Chern-Simons term in the action, which may contain a new form field strength $F_{n_{B}}$. The dilaton coupling $a_{B}$ of the new form field strength is then again fixed modulo its sign by the necessary condition $\bar{q}^{\left(e_{B}, m_{B}\right)}=1$. The intersection rules between the extremal branes corresponding to the different forms can be calculated and this fixes the relative signs of the dilaton couplings. We can then check if new openings are possible. If it is the case, we iterate the procedure until consistency of all the openings are ensured. In this way, we are able to reconstruct all the maximally oxidised theories

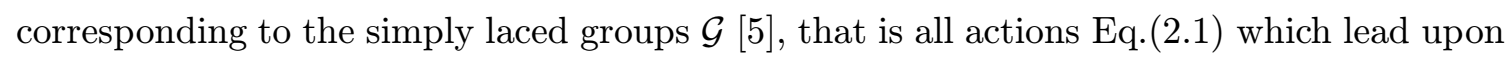
dimensional reduction to such group $\mathcal{G}$ and which are not dimensional reduction from an

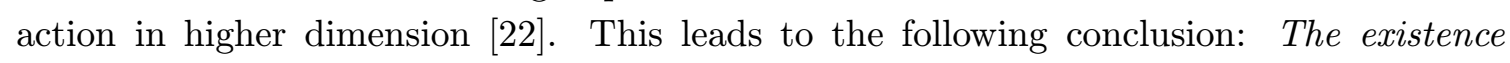
of BPS configurations with $\bar{q}=1$ between any electric extremal $p$-brane $(p \geq 1)$ and its magnetic dual, along with the requirement of consistency of brane opening in the original uncompactified theory (characterised by at most one dilaton), is a necessary and sufficient condition to have a theory whose dimensional reduction down to three dimensions has a simple simply laced group $\mathcal{G}$ symmetry.

Using Eq.(4.12) the condition $\bar{q}^{\left(e_{B}, m_{B}\right)}=1$ yields up to a sign the dilaton coupling

$$
a_{n}^{2}(D)=2 \frac{(1-q) D+\left(q^{2}+4 q-1\right)}{D-2} .
$$

with

$$
a_{n}^{2} \geq 0 \text {. }
$$

The procedure outlined above gives the following relations between $q, D$, the Chern-Simons terms, and the Lie group symmetry of the theory reduced to three dimensions exhibited in Table I 


\begin{tabular}{||c||c||c||l||l||c||}
\hline$q$ & $D$ & $a_{q+2}^{2}$ & Openings & \multicolumn{1}{|c}{$C . S}$. & $\mathcal{G}$ \\
\hline \hline 0 & - & $2(D-1) /(D-2)$ & - & - & $A_{D-1}$ \\
\hline 1 & - & $8 /(D-2)$ & - & - & $D_{D-2}$ \\
\hline 2 & 11 & 0 & $2 \cap 5=1$ & $A_{3} \wedge F_{4} \wedge F_{4}$ & $E_{8}$ \\
\hline 2 & 10 & $1 / 4$ & $2 \cap 4=1, \ldots$ & $A_{3} \wedge F_{4} \wedge H_{3}, \ldots$ & $E_{8}$ \\
\hline 2 & 9 & $4 / 7$ & $2 \cap 3=1$ & $A_{3} \wedge F_{4} \wedge F_{2}$ & $E_{7}$ \\
\hline 2 & 8 & 1 & $2 \cap \overline{2}=1$ & $A_{3} \wedge F_{4} \wedge F_{1}$ & $E_{6}$ \\
\hline 3 & 10 & 0 & - & - & $E_{7}$ \\
\hline
\end{tabular}

Table I : Reconstruction from branes of the simple simply laced Lie groups $G$.

We see indeed that all simple simply laced Lie groups (in their maximally non compact form) are recovered from the intersecting extremal brane solutions and their openings from

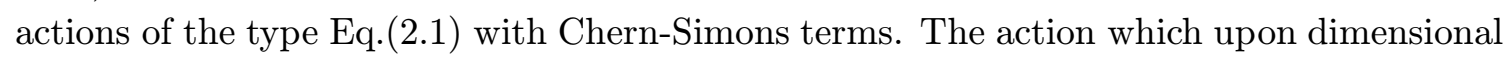
reduction to three dimensions exhibit these Lie group symmetry are fully determined.

\subsection{From $\mathcal{G}$ to $\mathcal{G}^{+++}$}

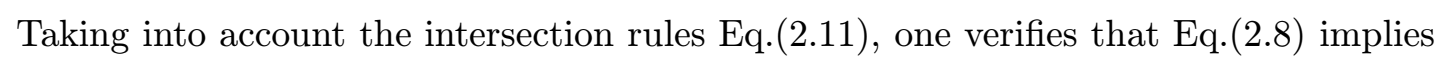

$$
\sum_{\alpha=1}^{D}\left(p^{(\alpha)}\right)^{2}-\frac{1}{2}\left(\sum_{\alpha=1}^{D} p^{(\alpha)}\right)^{2}+\frac{1}{2} \phi^{2}=\frac{D-2}{\Delta} \sum_{A} \ln ^{2} H_{A}\left(x^{a}\right),
$$

and we recall that the extremal branes in the intersecting brane configurations satisfy the

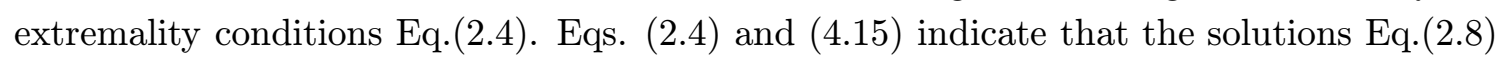
carry a group-theoretical significance in the triple (or very-) Kac-Moody extension $\mathcal{G}^{+++}$ of $\mathcal{G}$, for all simple groups $\mathcal{G}^{+++}$(simply laced or not) as we now show.

We first recall how all simple Lie algebra $\mathcal{G}$ can be embedded in a very-extended KacMoody algebra $\mathcal{G}^{+++}$. The simple roots of $\mathcal{G}^{+++}$are given by adding two nodes to the gravity line of the Dynkin diagram of the affine extension $\mathcal{G}^{+}$of $\mathcal{G}$, thus increasing by three the rank of $\mathcal{G}$ [23i]. The resulting Dynkin diagrams for $\mathcal{G}^{+++}$are shown in Fig.3. As in the case of Lie algebras, the Dynkin diagrams yield the Cartan matrix of $\mathcal{G}^{+++}$which is then entirely determined from the Serre relations.

The group $S L(D)$ defined by this triple extended gravity line can be extended to the full deformation group $G L(D)$ whose algebra, generated by $D^{2}$ generators $K_{b}^{a}, a, b=1, \ldots, D$ , is a subalgebra of $\mathcal{G}^{+++}$. The $K_{b}^{a}$ satisfy the following commutation relations

$$
\left[K_{b}^{a}, K_{d}^{c}\right]=\delta_{b}^{c} K_{d}^{a}-\delta_{d}^{a} K_{b}^{c} .
$$

One considers the Cartan subalgebra of $\mathcal{G}^{+++}$generated by the $K_{a}^{a}$ and $s=r-D$ abelian generators $R_{u}$ where $r$ is the rank of $\mathcal{G}^{+++}$. We write the corresponding abelian group element $\mathcal{V}_{\text {abelian }}$ as

$$
\mathcal{V}_{\text {abelian }}=\exp \left(-\sum_{a=1}^{D} p^{(a)} K_{a}^{a}-\sum_{u=1}^{s} \phi^{u} R_{u}\right)
$$




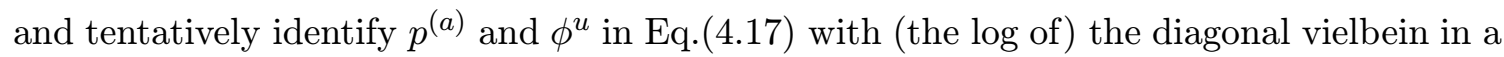
triangular gauge ${ }^{6} e_{\alpha}^{a}(x), \alpha \leq a$ and $\phi^{u}$ with dilaton fields $\phi^{u}(x)$ in any action $\widetilde{S}$ describing a maximally oxidised theory ${ }^{7}$. The actions $\widetilde{S}$ generalise to all simple Lie groups the ones reconstructed in Section 3 for simply laced groups. It takes the form

$$
\widetilde{S}=\frac{1}{16 \pi G_{N}^{(D)}} \int d^{D} x \sqrt{-g}\left[R-\frac{1}{2} \sum_{u=1}^{s}\left(\partial \phi^{u}\right)^{2}-\frac{1}{2} \sum_{I} \frac{1}{p_{I} !} \exp \left(\sum_{u=1}^{s} a_{I}^{u} \phi^{u}\right) F_{p_{I}}^{2}\right]+C . S .,
$$

where C.S. represents Chern-Simons terms that are required for some groups $\mathcal{G}$.
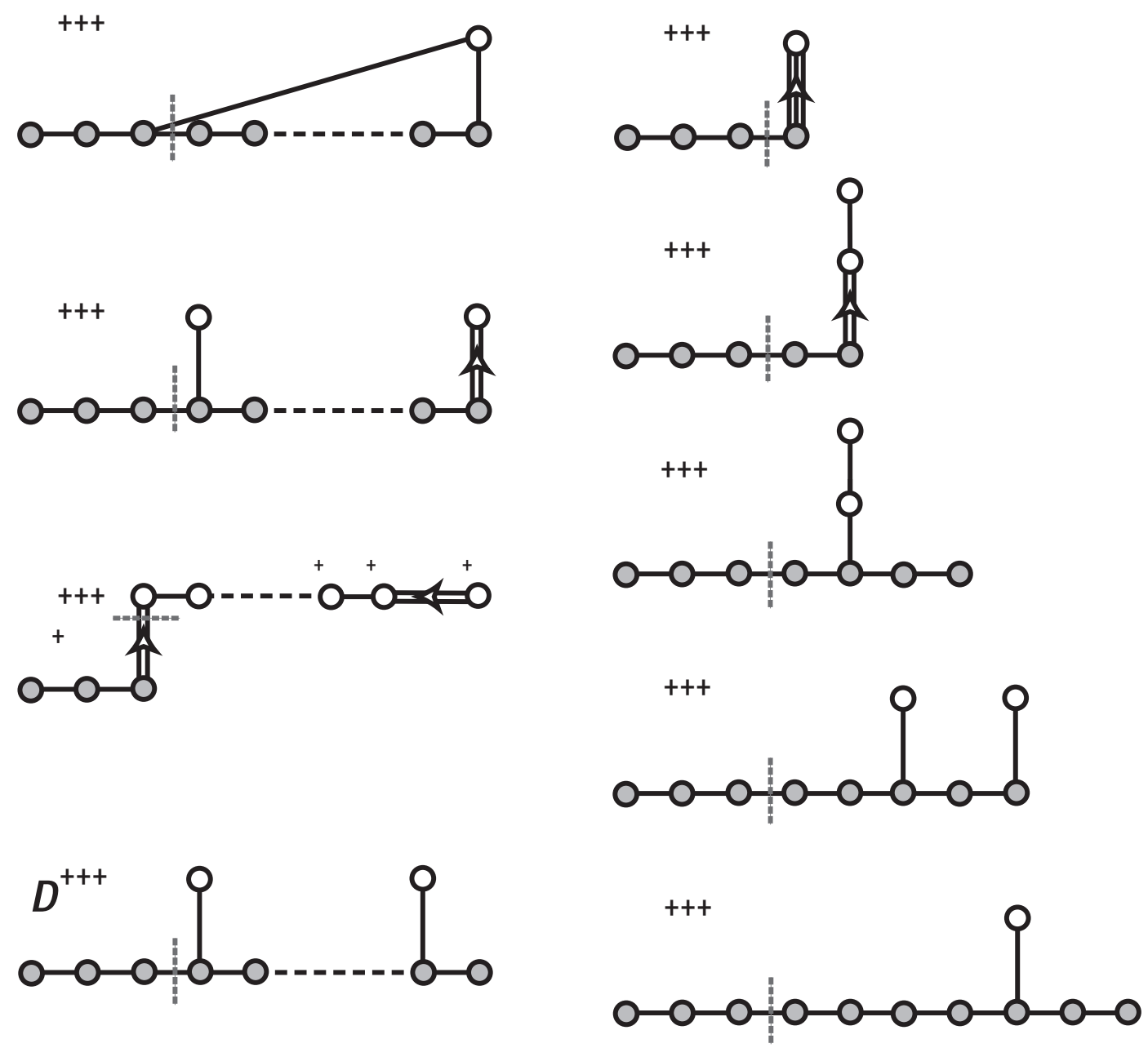

Fig.3. Dynkin diagram of $\mathcal{G}^{+++}$.

The nodes of the gravity line are shaded. The Dynkin diagram of $\mathcal{G}$ is that part of the diagram of $\mathcal{G}^{+++}$which sits on the right of the dashed line. The first three nodes define the Kac-Moody extensions.

\footnotetext{
${ }^{6}$ We can indifferently label the $p^{(a)}$ by a curved or a flat index, as it is uniquely defined by the diagonal vielbein in the triangular gauge. The position of this index as a subscript or superscript is a matter of convention and has no tensor significance.

${ }^{7} \mathrm{~A}$ geometrical motivation of this identification is given in $\left[\mathbb{8}^{3}\right]$ and an alternate motivation has been proposed in [24] by extending $G L(D)$ to $I G L(D)$. In this work the justification of the identification follows from the analysis of Section 5.
} 
Given any symmetrisable Kac-Moody algebra there exists, up to a numerical factor, a unique scalar product defined on the algebra that is invariant under the adjoint action of the algebra [2] $\overline{2}_{1}$. For a finite-dimensional simple Lie algebra this is just the Killing form which can be expressed as the trace of the generators in any finite-dimensional representation. In the Cartan subalgebra described in the $L_{i}=\left\{K_{a}^{a}, R_{u}\right\}$ basis it is $[\bar{i}]$

$$
\left\langle K^{a}{ }_{a}, K_{b}^{b}\right\rangle=\delta_{a b}-\frac{1}{2} \boldsymbol{\Xi}_{a b} \quad ; \quad\left\langle R_{u}, R_{v}\right\rangle=\frac{1}{2} \delta_{u v} \quad ; \quad\left\langle K_{a}^{a}, R_{v}\right\rangle=0,
$$

where $\boldsymbol{\Xi}$ is the matrix with all entries equal to one. The matrix $\mathbf{G}_{i j}=\left\langle L_{i}, L_{j}\right\rangle$ is invariant under the group of Weyl transformations (and of outer automorphisms) of $\mathcal{G}^{+++}$. Hence the quadratic form

$$
\sum_{\alpha=1}^{D}\left(p^{(\alpha)}\right)^{2}-\frac{1}{2}\left(\sum_{\alpha=1}^{D} p^{(\alpha)}\right)^{2}+\frac{1}{2} \sum_{u=1}^{s}\left(\phi^{u}\right)^{2}
$$

is invariant under the Weyl reflections in $\mathcal{G}^{+++}$.

Eq. $(4.20)$ is identical with the left hand side of Eq.(4.15). In addition, one can prove that the extremality condition Eq.(2.4) defines a a Weyl preserving embedding of $\mathcal{G}^{p+1}$ in $\mathcal{G}^{+++}$where $\mathcal{G}^{p+1}$ is the symmetry group resulting from dimensional reduction of the action Eq.(4.18i) to $(D-p-1)$ dimensions where the compactification is performed on a spatial $p$-torus and on time. These features would provide a strong indication of the existence of a hidden $\mathcal{G}^{+++}$symmetry of the theory defined by Eq.(4.18) or by some generalisation there of, if the right hand side of Eq.(4.15) would share such group-theoretical significance. In the next section, we show, by construction of an explicit $\mathcal{G}^{+++}$invariant action, that this is indeed the case.

\section{Non-linear realisation of $\mathcal{G}^{+++}$}

This section is based on reference [i] $1 \overline{2}$ in.

\subsection{The temporal involution and the coset space $\mathcal{G}^{+++} / K^{+++}$}

We want ultimately $\mathcal{G}^{+++}$to fully characterise the symmetry of the action $\widetilde{S}$ defined by Eq.(4.18]), or of some more general theory. As an enlargement of the global symmetry group $\mathcal{G}$ arising in the dimensional reduction of $\widetilde{S}, \mathcal{G}^{+++}$should also define a global symmetry. This poses a dilemma. The metric and the form field strengths in $\widetilde{S}$ are genuine space-time fields and $\widetilde{S}$ is invariant under the local diffeomorphism and gauge groups. How could a global symmetry encompass a local symmetry? The present analysis is an attempt to solve this dilemma by taking advantage of the infinite dimensionality of the algebra $\mathcal{G}^{+++}$. More precisely we replace the action $\widetilde{S}$ by an action $\mathcal{S}$ explicitly invariant under the global $\mathcal{G}^{+++}$ symmetry. $\mathcal{S}$ contains an infinite number of objects that are tensors under $S L(D)$. These comprise a symmetric tensor $g_{\mu \nu}$, scalars $\phi^{u}$ and $\left(p_{I}-1\right)$-form potentials $A_{\mu_{1} \mu_{2} \ldots \mu_{p_{I}-1}}$ which can be interpreted as the corresponding fields occurring in Eq.(4.18i) taken at a fixed space time point. Their motion in space-time, as well as those of possible additional fields, is expected to take place through an infinite number of field derivatives at this point, encoded in other objects in $\mathcal{S}$. 
The positive (negative) step operators in the $A_{D-1}$ subalgebra are, from Eq.(4.16), the $K^{a}{ }_{b}$ with $b>a(b<a)$. They define the level zero step operators of the $\mathcal{G}^{+++}$adjoint representation. The positive (negative) levels of the adjoint representation of $\mathcal{G}^{+++}$are defined as follows. One takes a set of $q$ non-negative (non-positive) integers, excluding $q$ zeros, where $q$ is the number of simple roots of $\mathcal{G}^{+++}$not contained in the gravity line. The $q$ integers count the number of times each such root appears in the decomposition of the adjoint representation of $\mathcal{G}^{+++}$into irreducible representations of $A_{D-1}$. Positive (negative) levels contain only positive (negative) roots and the number of irreducible representations of $A_{D-1}$ at each level is finite. All step operators of level greater than zero may be written as irreducible tensors $R_{d_{1} \ldots d_{s}}{ }^{{ }_{1} \ldots c_{r}}$ of the $A_{D-1}$ subalgebra of $\mathcal{G}^{+++}$, namely

$$
\left[K_{b}^{a}, R_{d_{1} \ldots d_{s}}^{{ }^{1} \ldots c_{r}}\right]=\delta_{b}^{c_{1}} R_{d_{1} \ldots d_{s}}^{a \ldots c_{r}}+\ldots+\delta_{b}^{c_{r}} R_{d_{1} \ldots d_{s}}^{{ }^{1} \ldots a}-\delta_{d_{1}}^{a} R_{b \ldots d_{s}}{ }^{c_{1} \ldots c_{r}}-\ldots-\delta_{d_{s}}^{a} R_{d_{1} \ldots b}{ }^{c_{1} \ldots c_{r}}
$$

The commutators of all positive step operators are generated by the commutators of step operators corresponding to simple roots. At level zero these 'simple step operators' are, from Eq. (4.1.16), the $K_{a+1}^{a}(a=1,2, \ldots, D-1)$. In general, when $s$ dilatons are present in the action $\widetilde{S}$, the rank of $\mathcal{G}^{+++}$is $D+s$. The $s$ abelian generators $R_{u}$ of its subgroup $G L(D) \times U(1)^{s}$ have non vanishing commutators with the tensor step operators $R^{a_{1} a_{2} \ldots a_{r}}$ associated to electric or magnetic simple roots. In dimensional reduction, these arise from $n$-form potentials where $n=p_{I}-1$ for an electric root and $n=D-p_{I}-1$ for a magnetic one. We can read off their commutation relation, in the normalisation given for the dilaton

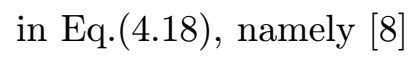

$$
\left[R_{u}, R^{a_{1} a_{2} \ldots a_{r}}\right]=-\varepsilon \frac{a_{I}^{u}}{2} R^{a_{1} a_{2} \ldots a_{r}},
$$

where $\varepsilon=+1$ for an electric root and -1 for a magnetic one.

To switch from positive $K_{b}^{a}(b>a)$ step operators to negative ones it suffices to interchange upper and lower indices. At higher levels, the negative of the tensor $R_{d_{1} \ldots d_{s}} c_{1 \ldots}$ is similarly a tensor $\bar{R}_{c_{1} \ldots c_{r}} d_{1 \ldots}$. The invariant scalar product for step operators is given by

$$
\left\langle K_{a}^{b}, K_{c}^{d}\right\rangle=\delta_{c}^{b} \delta_{a}^{d} a>b, d>c \quad ; \quad\left\langle R_{b_{1} \ldots b_{s}}^{a_{1} \ldots a_{r}}, \bar{R}_{d_{1} \ldots d_{r}}^{c_{1} \ldots c_{s}}\right\rangle=\delta_{b_{1}}^{c_{1}} \ldots \delta_{b_{s}}^{c_{s}} \delta_{d_{1}}^{a_{1}} \ldots \delta_{d_{r}}^{a_{r}}
$$

Iterative procedures to compute the step operators at any level can be devised. They build, together with the Cartan generators $K^{a}{ }_{a}(a=1,2, \ldots, D)$ and $R_{u}(u=1,2, \ldots, s)$, the full content of the adjoint representation of $\mathcal{G}^{+++}$.

The metric $g_{\mu \nu}$ at a fixed space time point parametrises the coset $G L(D) / S O(D-1,1)$. To construct a $\mathcal{G}^{+++}$invariant action $\mathcal{S}$ containing such a tensor, we shall build a non linear realisation of $\mathcal{G}^{+++}$in a coset space $\mathcal{G}^{+++} / K^{+++}$where the subgroup $K^{+++}$contains the Lorentz group $S O(D-1,1)$. We use a recursive construction based on the level decomposition of $\mathcal{G}^{+++}$. As at each level the $S O(D-1,1)$ invariance must be realised for a finite number of generators, we cannot use the Chevalley involution to build the coset $\mathcal{G}^{+++} / K^{+++}$. Rather we shall use a 'temporal' involution from which the required non-compact generators of $K^{+++}$can be selected.

The 'temporal' involution $\omega$ is defined in the following way. For the generators of the Cartan subalgebra we take, as in the Cartan involution,

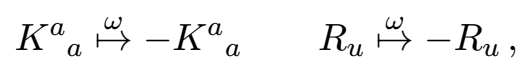


while for step operators we take

$$
R_{d_{1} \ldots d_{s}} c_{1} \ldots c_{r} \stackrel{\omega}{\mapsto}-\eta \bar{R}_{c_{1} \ldots c_{r}} d_{1} \ldots d_{s},
$$

with $\eta=(-1)^{n_{t}}$ where $n_{t}$ is the number of time indices in $R_{d_{1} \ldots d_{s}}{ }^{c_{1} \ldots c_{r}}$. All commutation

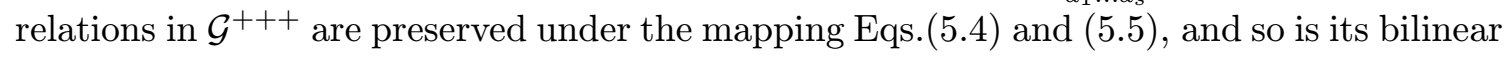
form. This mapping constitutes an involution that we label the temporal involution. We define the subgroup $K^{+++}$of $\mathcal{G}^{+++}$as the subgroup invariant under this involution. Its generators are

$$
R_{d_{1} \ldots d_{s}}^{c_{1} \ldots c_{r}}-\eta \bar{R}_{c_{1} \ldots c_{r}}{ }^{d_{1} \ldots d_{s}}
$$

$K^{+++}$contains the Lorentz group $S O(D-1,1)$ and all generators with $\eta=-1$ are noncompact .

\subsection{Non-linear realisation of $\mathcal{G}^{+++}$in $\mathcal{G}^{+++} / K^{+++}$}

We will follow a similar line of thought as the one developed in reference [1] of $E_{8}^{++}$. Consider a group element $\mathcal{V}$ built out of Cartan and positive step operators in $\mathcal{G}^{+++}$. It takes the form

$$
\mathcal{V}=\exp \left(\sum_{a \geq b} h_{b}{ }^{a} K_{a}^{b}-\sum_{u=1}^{s} \phi^{u} R_{u}\right) \exp \left(\sum \frac{1}{r ! s !} A_{b_{1} \ldots b_{s}}^{a_{1} \ldots a_{r}} R_{a_{1} \ldots a_{r}}^{b_{1} \ldots b_{s}}+\cdots\right)
$$

We have written it so that the first exponential contains only level zero operators (i.e the Cartan and the level zero positive step operators) and the second one contains the positive step operators of level strictly greater than zero. The tensors $h_{b}{ }^{a}, \phi^{u}, A_{b_{1} \ldots b_{s}} a_{1} \ldots a_{r}$, bear a priori no relation with the metric, the dilaton and the potentials of the $p_{I}$ form field strengths $F_{p_{I}}$ entering the action Eq.( $\left(\bar{A}_{1}-\overline{1}_{1}\right)$. However we shall see that a dictionary can be established relating the tensors which appear at low levels with the fields occurring in Eq. (4.18) at a fixed space-time point. For higher levels the dictionary between group parameters and space-time fields should arise from the analysis of the dynamics encoded in the $\mathcal{G}^{+++}$invariant action $\mathcal{S}$ below.

A differential motion in the coset $\mathcal{G}^{+++} / K^{+++}$can be constructed from Eq.(5.5. Define

$$
d v=d \mathcal{V} \mathcal{V}^{-1} \quad d \tilde{v}=-\omega d v \quad ; \quad d v_{\text {sym }}=\frac{1}{2}(d v+d \tilde{v})
$$

As $d v$ and $d \tilde{v}$ are differentials in the Lie algebra, $d v_{s y m}$ contains only the Cartan generators and the combinations of step operators $R_{d_{1} \ldots d_{s}}^{c_{1} \ldots c_{r}}+\eta \bar{R}_{c_{1} \ldots c_{r}}{ }_{d_{1} \ldots d_{s}}$.

To construct the action $\mathcal{S}$ we wish to map a manifold $\mathcal{M}$ into $\mathcal{G}^{+++}$. We do not want to take for $\mathcal{M}$ a space-time manifold, as this might require the explicit introduction of local symmetries which we hope to be hidden in the infinite algebra of $\mathcal{G}^{+++}$. We shall take for $\mathcal{M}$ a one-dimensional world-line in $\xi$-space, i.e. $d v_{\text {sym }}=d v_{\text {sym }}(\xi)=d \mathcal{V}(\xi) \mathcal{V}^{-1}(\xi)$, where $\mathcal{V}(\xi)$ are the group parameters appearing in Eq.( $\left(\overline{5}^{-}-\overline{7}_{1}\right)$ that are now fields dependent on the variable $\xi$. Here no connection is imposed a priori between $\xi$-space and space-time.

A reparametrisation invariant action is then

$$
\mathcal{S}=\int d \xi \frac{1}{n(\xi)}\left\langle\left(\frac{d v_{s y m}(\xi)}{d \xi}\right)^{2}\right\rangle
$$


where $n(\xi)$ is an arbitrary lapse function ensuring reparametrisation invariance on the world-line. The 'trace' \langle\rangle is given by Eqs.(14.1. $\left.\overline{9}_{1}^{\prime}\right)$ and $\left({ }_{5}^{5} . \overline{3}\right)$. It ensures the invariance of the non-linear action $\mathcal{S}$ defined on the coset space $\mathcal{G}^{+++} / K^{+++}$under global $\mathcal{G}^{+++}$ transformations.

We now compute the level zero of the action Eq.( $\left(5^{5} .9\right)$, that is the terms generated by

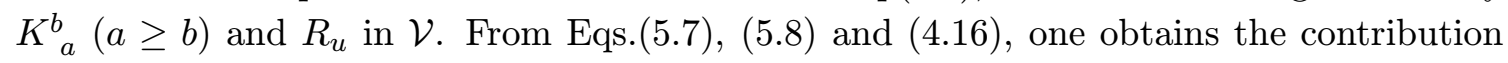
of the level zero to $v_{\text {sym }}(\xi)$,

$$
\frac{d v_{s y m}^{0}(\xi)}{d \xi}=-\frac{1}{2} \sum_{a \geq b}\left[e^{h}\left(\frac{d e^{-h}}{d \xi}\right)\right]_{b}{ }^{a}\left(K_{a}^{b}+\eta K_{b}^{a}\right)-\sum_{u=1}^{s} \frac{d \phi^{u}}{d \xi} R_{u}
$$

where $h$ is triangular matrix with elements $h_{b}{ }^{a}$. We now evaluate $\left\langle\left(d v_{\text {sym }}^{0}(\xi) / d \xi\right)^{2}\right\rangle$ using Eqs.(4.19i) and (15ing or lowering of the $a, b$ indices of the $\xi$-fields multiplying the negative step operator in $\left\langle d v_{\text {sym }}^{0} / d \xi, d v_{\text {sym }}^{0} / d \xi\right\rangle$ with the Minkowskian metric $\eta_{a b}$. This ensures that this expression is a Lorentz scalar. The Lorentz invariant action at level zero, $\mathcal{S}^{0}$, is

$$
\begin{aligned}
& \mathcal{S}^{(0)}= \int d \xi \frac{1}{n(\xi)}\left\langle\left(\frac{d v_{\text {sym }}^{0}(\xi)}{d \xi}\right)^{2}\right\rangle, \\
&=\frac{1}{2} \int d \xi \frac{1}{n(\xi)}\left\{\left[e^{h}\left(\frac{d e^{-h}}{d \xi}\right)\right]_{b}{ }^{a}\left[e^{h}\left(\frac{d e^{-h}}{d \xi}\right)\right]_{a}^{T} b+\left[e^{h}\left(\frac{d e^{-h}}{d \xi}\right)\right]_{b}{ }^{a}\left[e^{h}\left(\frac{d e^{-h}}{d \xi}\right)\right]_{a}^{b}\right. \\
&\left.-\left(\left[e^{h}\left(\frac{d e^{-h}}{d \xi}\right)\right]_{a}^{a}\right)^{2}+\sum_{u=1}^{s}\left(\frac{d \phi^{u}}{d \xi}\right)^{2}\right\}
\end{aligned}
$$

where the summation is performed over Lorentz indices. Note that the lower indices of $e^{-h}$ and the upper indices of $e^{h}$ cannot be lowered or raised by the Lorentz metric. To avoid confusion we label these indices with greek letters, namely we define 'vielbein'

$$
e_{\mu}^{a}=\left(e^{-h}\right)_{\mu}^{a} \quad e_{b}^{\mu}=\left(e^{h}\right)_{b}^{\mu} \quad ; \quad g_{\mu \nu}=e_{\mu}^{a} e_{\nu}^{b} \eta_{a b} .
$$

Although we have not yet introduced a space-time, we shall name the $a$ indices flat and the $\mu$ indices curved. As a result of the temporal involution and of the scalar product \langle\rangle in $\mathcal{G}^{+++}$, the flat-index tensors have been endowed with a Lorentz metric while curved-index tensors define a metric in $G L(D) / S O(D-1,1)$. Hence, for any $\xi$, we are allowed to identify $g_{\mu \nu}(\xi)$ in Eq. $(\overline{5} .12 i)$ as the metric tensor in $\widetilde{S}$, Eq. (4.18i), at a fixed space-time point.

Using Eq.(5.121), one can rewrite the action Eq.(5.11) as

$$
\mathcal{S}^{(0)}=\frac{1}{2} \int d \xi \frac{1}{n(\xi)}\left[\frac{1}{2}\left(g^{\mu \nu} g^{\sigma \tau}-\frac{1}{2} g^{\mu \sigma} g^{\nu \tau}\right) \frac{d g_{\mu \sigma}}{d \xi} \frac{d g_{\nu \tau}}{d \xi}+\sum_{u=1}^{s} \frac{d \phi^{u}}{d \xi} \frac{d \phi^{u}}{d \xi}\right]
$$

At higher levels, the tensors multiplying the step operators couple nonlinearly to the level zero objects and between themselves. The coupling to the metric and to $\phi^{u}$ can be formally written down for all levels, but the self-coupling of the $A_{b_{1} \ldots b_{s}} \underset{a_{1} \ldots a_{r}}{ }$ depend specifically on the group $\mathcal{G}$. 
Consider a general $A_{D-1}$ tensor $A_{a_{1} \ldots a_{r}} b_{1} \ldots b_{s}$ parametrising a normalised step operator $R_{b_{1} \ldots b_{s}} \begin{gathered}a_{1} \ldots a_{r} \\ \text {. The commutation relations of } R_{b_{1} \ldots b_{s}} a_{1} \ldots a_{r}\end{gathered}$ with the $K^{b}{ }_{a}$ are given by the tensor transformations as in Eq. (4.

$$
\left[R_{u}, R_{b_{1} \ldots b_{s}}^{a_{1} \ldots a_{r}}\right]=\lambda_{u} R_{b_{1} \ldots b_{s}}^{a_{1} \ldots a_{r}}
$$

Here $\lambda_{u}=\sum \lambda_{u, i}$ where the $\lambda_{u, i}$ are the scale parameters of the simple step operators entering the multiple commutators defining $R_{b_{1} \ldots b_{s}} a_{1} \ldots a_{r}$. This property follows from the Jacobi identity. Identifying for simple step operators $\lambda_{u, i}$ with $-\varepsilon a_{I}^{u} / 2$ in Eq.(5.5.2.

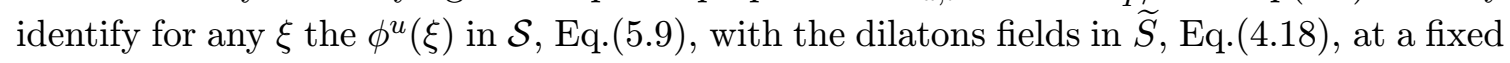
space time point. The particular $A_{a_{1} \ldots a_{r}} b_{1} \ldots b_{s}(\xi)$ multiplying the step operators belonging to the subgroup $\mathcal{G}$ can be similarly identified to the corresponding potential forms in $\widetilde{S}$ along with their duals.

It is straightforward to compute the contribution $d v^{(A)}$ to $d v$ of a given tensor when commutators of the $R_{b_{1} \ldots b_{s}}^{a_{1} \ldots a_{r}}$ between themselves are disregarded. On gets

$$
d v^{(A)}=\frac{1}{r ! s !} d A_{\mu_{1} \ldots \mu_{r}}^{\nu_{1} \ldots \nu_{s}} \exp \left(-\sum_{u=1}^{s} \lambda^{u} \phi^{u}\right) e_{a_{1}}^{\mu_{1}} \ldots e_{a_{r}}^{\mu_{r}} e_{\nu_{1}}^{b_{1}} \ldots e_{\nu_{s}}^{b_{s}} R_{b_{1} \ldots b_{s}}^{a_{1} \ldots a_{r}}
$$

The contribution $\mathcal{S}_{0}^{(A)}$ of $v^{(A)}$ to the action $\mathcal{S}$ is computed as previously and one gets

$\mathcal{S}_{0}^{(A)}=\frac{1}{2} \int d \xi \frac{1}{n(\xi)}\left[\frac{1}{r ! s !} \exp \left(-\sum_{u=1}^{s} 2 \lambda^{u} \phi^{u}\right) \frac{d A_{\mu_{1} \ldots \mu_{r}}^{\nu_{1} \ldots \nu_{s}}}{d \xi} g^{\mu_{1} \mu_{1}^{\prime} \ldots g^{\mu_{r} \mu_{r}^{\prime}}} g_{\nu_{1} \nu_{1}^{\prime} \ldots g_{\nu_{s} \nu_{s}^{\prime}}} \frac{d A_{\mu_{1}^{\prime} \ldots \mu_{r}^{\prime}} \nu_{\nu_{s}^{\prime}}^{\prime}}{d \xi}\right]$.

The full action can only be approached in a recursive way. In $\mathcal{S}_{0}^{(A)}$, one must replace derivatives by non linear generalisations to take into account the non vanishing commutators between tensor step operators. We represent such terms by 'covariant' derivatives

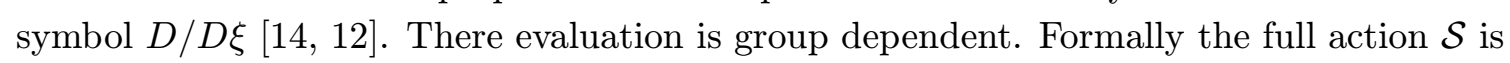

$$
\mathcal{S}=\mathcal{S}^{(0)}+\sum_{A} \mathcal{S}^{(A)}
$$

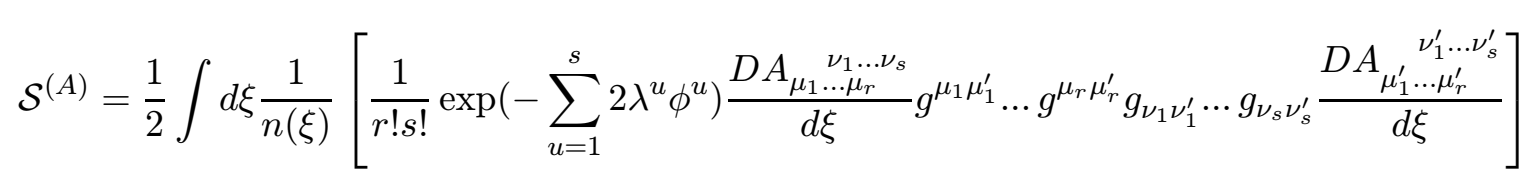
where the sum on $A$ is a summation over all tensors appearing at all positive levels in the decomposition of $\mathcal{G}^{+++}$into irreducible representations of $A_{D-1}$.

One may expand $\mathcal{S}$ given in Eq.(5.17) in power of fields parametrising the positive step operators. Up to quadratic terms, the result $\mathcal{S}^{(Q)}$ is obtained by retaining in $v(\xi)$ terms independent or linear in these fields. Define the one-forms $[d A]$ and the moduli $p^{(a)}$ (or $\left.p^{(\mu)}\right)$ as in Section 4.3 by

$$
\begin{gathered}
\exp \left(-\sum_{u=1}^{s} \lambda^{u} \phi^{u}\right) \hat{e}_{a_{1}}^{\mu_{1}} \ldots \hat{e}_{a_{r}}^{\mu_{r}} \hat{e}_{\nu_{1}}^{b_{1}} \ldots \hat{e}_{\nu_{s}}^{b_{s}} \frac{d A_{\mu_{1} \ldots \mu_{r}}^{\nu_{1} \ldots \nu_{s}}}{d \xi} \stackrel{\operatorname{def}}{=} \frac{[d A]_{a_{1} \ldots b_{r} \ldots b_{s}}}{d \xi} \\
\hat{e}_{b}{ }^{\mu} \frac{d \hat{e}_{\mu}{ }^{a}}{d \xi} \stackrel{\text { def }}{=} \frac{d p^{(a)}}{d \xi}
\end{gathered}
$$


where $\hat{e}$ means that only the diagonal vielbein are kept. We get

$$
\begin{aligned}
\mathcal{S}^{(Q)}= & \int d \xi \frac{1}{n(\xi)}\left[\sum_{a=1}^{D}\left(\frac{d p^{(a)}}{d \xi}\right)^{2}-\frac{1}{2}\left(\sum_{a=1}^{D} \frac{d p^{(a)}}{d \xi}\right)^{2}+\frac{1}{2} \sum_{u=1}^{s}\left(\frac{d \phi^{u}}{d \xi}\right)^{2}\right. \\
& \left.+\frac{1}{2}\left(e_{b}{ }^{\mu} \frac{d e_{\mu}^{a}}{d \xi} \frac{d e_{a \nu}}{d \xi} e^{\nu b}\right)^{(1)}+\frac{1}{2} \frac{1}{r ! s !} \sum_{A} \frac{[d A]_{a_{1} \ldots a_{r}} \ldots b_{s}}{d \xi} \frac{[d A]_{b_{1} \ldots b_{s}} a_{a_{1} \ldots a_{r}}}{d \xi}\right],
\end{aligned}
$$

where the superscript (1) in the vielbein term indicates that only terms quadratic in $h_{b}{ }^{a}(a>b)$ are kept. In the next section we shall produce solutions of the action Eq. $(5.20)$ which are exact solutions of the full action Eq.(5.17i).

\section{Extremal branes from the $\mathcal{G}^{+++}$invariant actions}

We shall look for solutions of the equations of motion derived from $\mathcal{S}$ and containing only one $A(\xi)$-field, or one non-diagonal $h(\xi)$-field, with given indices [1]2근. Such truncation is consistent with all the equations of motion and hence we may disregard all non-linearity in the step operators. Therefore it suffices for obtaining such solutions to replace the action $\mathcal{S}$ by its simplified version Eq. $(\overline{5} \cdot \overline{5} \cdot \overline{0})$.

We shall as in Eqs. $\left({ }_{2}^{2} .5 i\right)$ and $(2.6)$, consider $A$ to be an antisymmetric tensor with a time index $\tau$ and $r$ space indices coupled to a step operator of the $\mathcal{G}$ subalgebra. The equation of motions are

a) The lapse constraint.

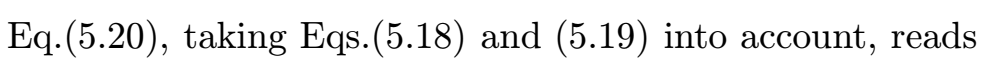

$$
\left.\sum_{\alpha=1}^{D}\left(\frac{d p^{(\alpha)}}{d \xi}\right)^{2}-\frac{1}{2}\left(\sum_{\alpha=1}^{D} \frac{d p^{(\alpha)}}{d \xi}\right)^{2}+\frac{1}{2}\left(\frac{d \phi}{d \xi}\right)^{2}-\frac{1}{2} \exp \left[\varepsilon a \phi-2 p^{(\tau)}-2 \sum_{\lambda=\lambda_{1}}^{\lambda_{r}} p^{(\lambda)}\right)\right]\left(\frac{d A_{\tau \lambda_{1} \ldots \lambda_{r}}}{d \xi}\right)^{2}=0
$$

Here we have taken one dilaton with scaling $\lambda=-\varepsilon a / 2$ in accordance with Eq.(管). Note that this relation is valid wether or not the magnetic root is simple, as seen in dimensional reduction. A crucial feature of this equation is the minus sign in front of the exponential. Its origin can be traced back to the temporal involution defining our coset space, hence to Lorentz invariance, because both magnetic and electric potentials have a time index.

b) The equation of motion for $A$.

We take the lapse $n(\xi)=1$. One gets

$$
\frac{d}{d \xi}\left(\exp \left[\varepsilon a \phi-2 p^{(\tau)}-2 \sum_{\lambda=\lambda_{1}}^{\lambda_{r}} p^{(\lambda)}\right] \frac{d A_{\tau \lambda_{1} \ldots \lambda_{r}}}{d \xi}\right)=0 .
$$

c) The dilaton equation of motion.

$$
-\frac{d^{2} \phi}{d \xi^{2}}-\frac{1}{2} \varepsilon a \exp \left[\varepsilon a \phi-2 p^{(\tau)}-2 \sum_{\lambda=\lambda_{1}}^{\lambda_{r}} p^{(\lambda)}\right]\left(\frac{d A_{\tau \lambda_{1} \ldots \lambda_{r}}}{d \xi}\right)^{2}=0 .
$$


d) The vielbein equations of motion.

$$
\begin{gathered}
-2 \frac{d^{2} p^{(\alpha)}}{d \xi^{2}}+\sum_{\beta=1}^{D} \frac{d^{2} p^{(\beta)}}{d \xi^{2}}=0 \quad \alpha \neq \tau, \lambda_{i} \quad(i=1,2 \ldots r) \\
-2 \frac{d^{2} p^{(\alpha)}}{d \xi^{2}}+\sum_{\beta=1}^{D} \frac{d^{2} p^{(\beta)}}{d \xi^{2}}+\exp \left[\varepsilon a \phi-2 p^{(\tau)}-2 \sum_{\lambda=\lambda_{1}}^{\lambda_{r}} p^{(\lambda)}\right]\left(\frac{d A_{\tau \lambda_{1} \ldots \lambda_{r}}}{d \xi}\right)^{2}=0 \quad \alpha=\tau, \lambda_{i} .
\end{gathered}
$$

We take as anzätze the solutions of the extremal brane problem but with $H_{A}$ an unknown function of $H(\xi)$. Namely we pose

$$
\begin{aligned}
A_{\tau \lambda_{1} \ldots \lambda_{r}} & =\epsilon_{\tau \lambda_{1} \ldots \lambda_{r}}\left[\frac{2(D-2)}{\Delta}\right]^{1 / 2} H^{-1}(\xi), \\
p^{(\tau)}=p^{\left(\lambda_{i}\right)}=-\frac{D-r-3}{\Delta} \ln H(\xi) & ; \quad p^{(\alpha)}=\frac{r+1}{\Delta} \ln H(\xi) \quad \alpha \neq \tau, \lambda_{i} . \\
\phi & =\frac{D-2}{\Delta} \varepsilon a \ln H(\xi) .
\end{aligned}
$$

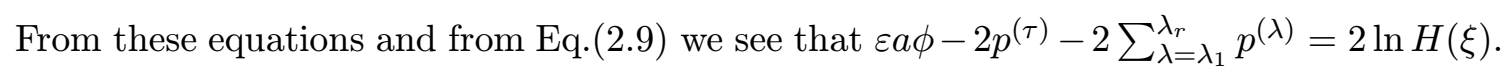

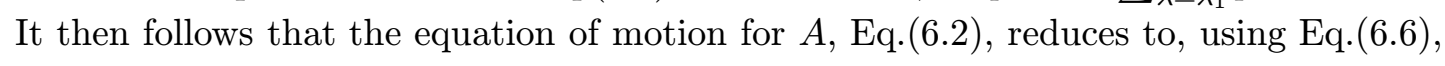

$$
\frac{d^{2} H(\xi)}{d \xi^{2}}=0
$$

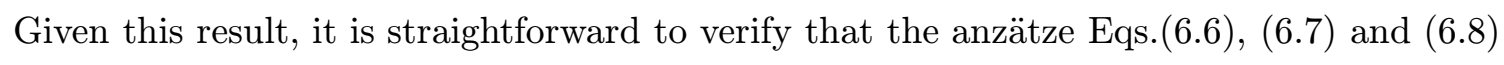
satisfy the dilaton and the vielbein equations of motions. The lapse constraint takes the form

$$
\sum_{\alpha=1}^{D}\left(d p^{(\alpha)}\right)^{2}-\frac{1}{2}\left(\sum_{\alpha=1}^{D} d p^{(\alpha)}\right)^{2}+\frac{1}{2}(d \phi)^{2}-\frac{D-2}{\Delta}(d \ln H)^{2}=0 .
$$

where the differentials are taken in $\xi$-space. It has therefore exactly the same form in

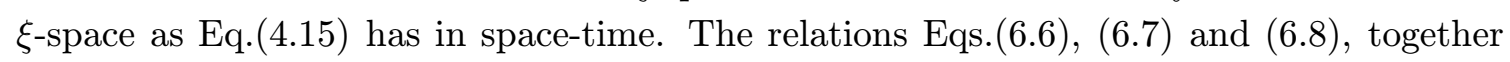

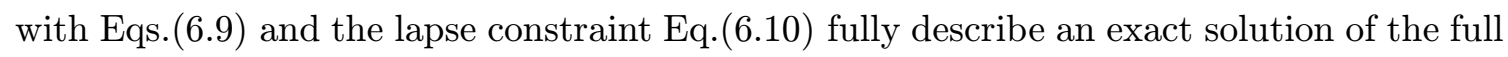

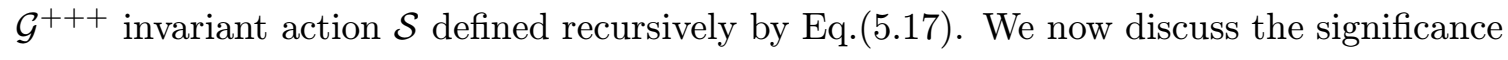
of this result.

The Eqs. $(\overline{6} \cdot \overline{6}),(\overline{6} . \overline{1})$ and $(\overline{6} . \overline{8})$ characterise completely the algebraic structure of the extremal brane solution but do not yield its harmonic character in space-time. As the functions $A_{\tau \lambda_{1} \ldots \lambda_{r}}(\xi), p^{(\tau)}(\xi), p^{\left(\lambda_{i}\right)}(\xi)$ and $\phi(\xi)$ were interpreted in the action $\mathcal{S}$ as functions at a fixed space-time point of the independent variable $\xi$, this is a consistent result. The solution $H=a+b \xi$ of Eq. $\left(\overline{6}_{1} \cdot \overline{9}_{i}^{\prime}\right)$ would then describe a motion in the space of solutions, for instance of branes with different charges. However the fact that we have exact solutions of the action $\mathcal{S}$ with the correct algebraic structure of the extremal branes, means that these solutions are only indirectly related to the corresponding space-time solution. One expects that the information contained in this solution, which is of course not contained in a trivial constant space-time solution of the Einstein equation, is the required information to build 
coupled equations to higher space-time derivatives encoded in higher level representations, which would then be directly related to space-time solutions.

There are indications that this may indeed be the case but it is unclear whether it is sufficient to consider the adjoint representation of $\mathcal{G}^{+++}[1 \overline{2} \overline{2}]$ or if one has to include other representations $\left[\overline{2} \overline{2} \bar{\sigma}_{1}^{\prime}, \overline{2} \overline{2} \bar{Z}_{1}\right]$.

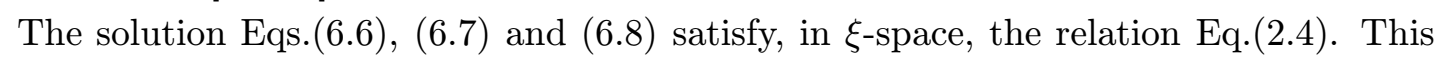
relation define an embedding of a subgroup $\mathcal{G}^{p+1}$ of $\mathcal{G}^{+++}$acting on the $p$ compact space dimensions in which the branes live and on the time dimension [iَin]. We shall consider the subgroup $\mathcal{G}^{p}$ of $\mathcal{G}^{p+1}$ which acts on the space dimensions only and we take $p \leq D-4$ so that $\mathcal{G}^{p+1}$ is a Lie group. This group is conjugate by a Weyl reflection in $\mathcal{G}^{+++}$of the group

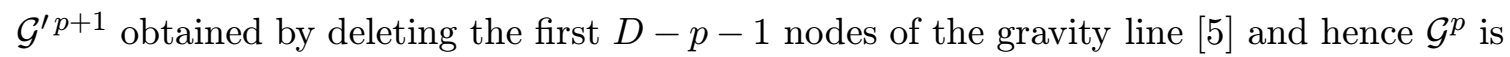
conjugate to its subgroup $\mathcal{G}^{p}$ characterising the usual dimensional reduction of Eq.(4.1. to $D-p$ dimensions.

We shall consider the transformations mapping one root to another root, thereby generating solutions of the same 'family' as the extremal solution just described. These transformations include the Weyl group $W\left(\mathcal{G}^{+++}\right)$of $\mathcal{G}^{+++}$. We shall examine Weyl transforms of the extremal brane solution characterised by one positive step operator which send the positive root into a positive root. Such transformations leave invariant not only $\mathcal{S}$ but also preserves their quadratic truncation Eq. $(5.20 \overline{0})$. Hence Eq. $(6.10)$ is invariant under the Weyl group of $\mathcal{G}^{p+1}$. The restriction to the Weyl group of $\mathcal{G}^{p}$ selects transformed fields with one time index.

Thus $W\left(\mathcal{G}^{p}\right)$ leaves invariant the quadratic form

$$
\sum_{a=1}^{D}\left(d p^{(a)}\right)^{2}+\frac{1}{2}\left[-\left(\sum_{i=1}^{D} d p^{(a)}\right)^{2}+\sum_{u=1}^{s}\left(d \phi^{u}\right)^{2}+\left(e_{b}^{\mu} d e_{\mu}^{a} d e_{a \nu} e^{\nu b}\right)^{(1)}+\frac{1}{r ! s !} \sum_{A}[d A]_{a_{1} \ldots a_{r}}^{b_{1} \ldots b_{s}}[d A]_{b_{1} \ldots b_{s}}^{a_{1} \ldots a_{r}}\right]
$$

and the embedding relation Eq.(2.2.4is $)$ in $\xi$-space. It acts on $A$-fields, or non-diagonal vielbein, containing one time index. The sum of the first three terms is the invariant metric of $\mathcal{G}^{+++}$

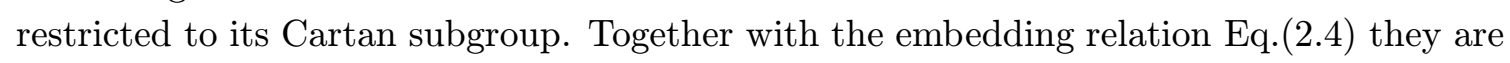
left invariant under the Weyl group of $\mathcal{G}^{p+1}$. We now see the group theoretical relevance

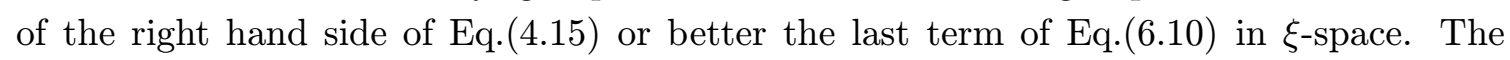
Weyl transformations of the step operators and the additional terms in Eq.(6.11) guarantee indeed the invariance of Eq. $\left(\overline{6}^{-} \cdot \overline{1}_{-}\right)$under $W\left(\mathcal{G}^{p}\right)$. For all $\mathcal{G}^{+++}$, the Weyl transformations generate new solutions from one extremal brane solution. We stress again that in the present approach both electric and magnetic branes are described 'electrically'.

For M-theory, this yield the well-known duality symmetries of M-theory, including the duality transformations of branes, KK waves and KK monopoles (Taub-NUT spaces). Such transformations are however not a privilege of M-theory and occur in all $\mathcal{G}^{+++}$invariant actions. This is exemplified below, taking for definiteness the action $\mathcal{S}$ for the group $E_{7}^{+++}$ which is related to the action $\widetilde{S}$ with gravity coupled to a 4- and a 2- form field strength in 9 space-time dimensions. The Dynkin diagram of $E_{7}^{+++}$is depicted in Fig.3, which exhibits the two simple electric roots (10) and (9) corresponding respectively to the step operators $R^{789}$ and $R^{9}$ which couple to the electric potentials $A_{789}$ and $A_{9}$. 
We take as input the electric extremal 2-brane $\mathbf{e}_{(8,9)}$ in the directions $(8,9)$ associated with the 4-form field strength whose corresponding potential is $A_{189}$ and submit it to the non trivial Weyl reflection $W_{10}$ associated with the electric root (10) of Fig.3. We display below, both for $\mathbf{e}_{(8,9)}$ and its transform, the moduli, i.e. the vielbein components $p^{(a)}$ and the the dilaton value $\phi$, of the brane solution Eqs.(6.7. vector where the last component is the dilaton. We also indicate the transform of the step operator $R^{189}$ under the Weyl transformation. We obtain

$$
\begin{array}{rrr}
(-4,3,3,3,3,3,3,-4,-4 ; 2 \sqrt{7}) \frac{\ln H(\xi)}{14} & \mathbf{e}_{(8,9)} & R^{189} \\
& \downarrow W_{10} & \\
(-7,0,0,0,0,0,7,0,0 ; 0) \frac{\ln H(\xi)}{14} & \mathbf{k k}_{\mathbf{e}(7)} & K_{7}^{1}
\end{array}
$$

The transformation of the 2-brane is reminiscent of a double T-duality in M-theory.

We now move the electric brane through Weyl reflections associated with roots of the gravity line to $\mathbf{e}_{(5,9)}$ and submit it to the Weyl reflection $W_{10}$. We now find that the brane $\mathbf{e}_{(5,9)}$ is invariant but moving it to the position $\mathbf{e}_{(5,6)}$, we get

$$
\begin{array}{ccc}
(-4,3,3,3,-4,-4,3,3,3 ; 2 \sqrt{7}) \frac{\ln H(\xi)}{14} & \begin{array}{c}
\mathbf{e}_{(5,6)} \\
\\
\downarrow W_{10}
\end{array} & R^{156} \\
(-1,6,6,6,-1,-1,-1,-1,-1 ; 4 \sqrt{7}) \frac{\ln H(\xi)}{14} & \mathbf{m}_{(5,6,7,8,9)} & R^{156789}
\end{array}
$$

This is a magnetic 5-brane in the directions $(5,6,7,8,9)$ associated to the 2 -form field strength! It is expressed in terms of its dual potential $A_{156789}$. Submit instead $\mathbf{e}_{(5,9)}$ to to the Weyl reflection $W_{9}$ associated with the electric root (9) of Fig.3. The 2-brane $\mathbf{e}_{(5,9)}$ is again invariant, but moving it to to the position $\mathbf{e}_{(5,6)}$, we now get

$$
\begin{aligned}
& \begin{array}{ccc}
(-4,3,3,3,-4,-4,3,3,3 ; 2 \sqrt{7}) \frac{\ln H(\xi)}{14} & \mathbf{e}_{(5,6)} \quad R^{156} \\
& \downarrow W_{9}
\end{array} \\
& (-3,4,4,4,-3,-3,4,4,-3 ;-2 \sqrt{7}) \frac{\ln H(\xi)}{14} \quad \mathbf{m}_{(5,6,9)} \quad R^{1569}
\end{aligned}
$$

This is a magnetic 3 -brane in the directions $(5,6,9)$ associated to the 4 -form field strength, expressed in terms of its dual potential $A_{1569}$.

Finally, let us submit the magnetic 5-brane $\mathbf{m}_{(5,6,7,8,9)}$ obtained in Eq.(6.15i) to the Weyl reflection $W_{9}$. One obtains

$$
\begin{aligned}
& \begin{array}{c}
(-1,6,6,6,-1,-1,-1,-1,-1 ; 4 \sqrt{7}) \frac{\ln H(\xi)}{14} \mathbf{m}_{(5,6,7,8,9)} \quad R^{156789} \\
\downarrow W_{9}
\end{array} \\
& (0,7,7,7,0,0,0,0,-7 ; 0) \frac{\ln H(\xi)}{14} \mathbf{k k}_{\mathbf{m}(2,3,4 ; 9)} R^{156789,9}
\end{aligned}
$$

Eq.(6.17) describes, as in M-theory, a purely gravitational configuration, namely a KKmonopole with transverse directions $(2,3,4)$ and Taub-NUT direction (9) in terms of a dual gravity tensor $h_{156789,9}$. 
It is also possible to generate solutions not contained, at least explicitly, in the group $\mathcal{G}^{p}$. These are very interesting solutions as they may test the significance of genuine KacMoody extensions of the Lie groups. Such analysis is outside the scope of the present work where we test only solutions which can straightforwardly be mapped to space time solutions of the effective actions Eq.(4.18i).

The above example illustrate the analogy of M-theory duality transformations with similar transformations in all 'M-theories' defined by all $\mathcal{G}^{+++}$. One may indeed carry the same analysis for all $\mathcal{G}^{+++}$and exhibit for each of them the 'duality' transformations of the branes. As in M-theory, these dualities are symmetries in non-compact space-time. This is because $\mathcal{G}^{p+1}$ is, as $\mathcal{G}^{p+1}$, the Lie group symmetry of the action Eq. (4.18) dimensionally reduced to three dimensions (for $p=D-4$ ). They differ because while the latter reduction leaves a Lorentzian non-compact space-time, the former leads to a Euclidean space-time by compactifying time. The group $\mathcal{G}^{p}$ of transformations on $\xi$-space discussed above, is thus in one to one correspondence with the group $\mathcal{G}^{p}$ of space-time transformations when time is decompactified. In particular, the functions $H(\xi)$ can thus be mapped into harmonic functions $H\left(\left\{x^{\nu}\right\}\right)$. However as pointed out in the previous section, more work is needed to relate directly $H(\xi)$ to $H\left(\left\{x^{\nu}\right\}\right)$, and solutions in $\xi$-space to solutions in space-time for all $\mathcal{G}^{+++}$, through translation operators hopefully induced by group generators.

\section{Acknowledgments}

This work was supported in part by the NATO grant PST.CLG.979008, by the "Actions de Recherche Concertées" of the "Direction de la Recherche Scientifique - Communauté Française de Belgique, by a "Pôle d'Attraction Interuniversitaire" (Belgium), by IISNBelgium (convention 4.4505.86), by Proyectos FONDECYT 1020629, 1020832 and 7020832 (Chile) and by the European Commission RTN programme HPRN-CT00131, in which F. E. and L. H. are associated to the Katholieke Universiteit te Leuven (Belgium).

\section{References}

[1] J. P. Gauntlett, Intersecting branes, Proceeding of the February 97 APCTP Winter School on Dualities of Gauge and String Theories, hep-th/9705011, and references therein.

[2] A. Strominger, Open p-branes, Phys. Lett. B383 (1996) 44, hep-th/9512059.

[3] P. K. Townsend, Brane surgery in Proceedings of the European Research Conference on 'Advanced Quantum Field Theory', La Londe les Maures, France, Sept. 1996, hep-th/9609207.

[4] R. Argurio, F. Englert, L. Houart and P. Windey, On the opening of branes, Phys. Lett. B408 (1997) 151.

[5] F. Englert, L. Houart and P. West, Intersection Rules, Dynamics and Symmetries, J. High Energy Phys. 08 (2003) 025, hep-th/0307024.

[6] P. West, $E_{11}$ and $M$ theory, Class. Quant. Grav. 18 (2001) 4443, hep-th/0104081.

[7] N. D. Lambert and P. C. West, Coset symmetries in dimensionally reduced bosonic string theory, Nucl. Phys. B615 (2001) 117, hep-th/0107209. 
[8] F. Englert, L. Houart, A. Taormina and P. West, The Symmetry of M-theories, J. High Energy Phys. 09 (2003) 020, hep-th/0304206.

[9] T. Damour, M. Henneaux and H. Nicolai, Cosmological billiards, Class. and Quant. Grav. 20 (2003) R145, hep-th/0212256, and references therein.

[10] T. Damour, M. Henneaux, E(10), BE(10) and arithmetical chaos in superstring cosmology, Phys. Rev.Lett. 86 (2001) 4749, hep-th/0012172; T. Damour, M. Henneaux, B. Julia and H. Nicolai, Hyperbolic Kac-Moody algebras and chaos in Kaluza-Klein models, Phys. Lett. B509 (2001) 323, hep-th/0103094.

[11] T. Damour, S. de Buyl, M. Henneaux and C. Schomblond, Einstein billiards and overextensions of finite-dimensional simple Lie algebras, J. High Energy Phys. 08 (2002) 030, hep-th/0206125.

[12] F. Englert and L. Houart, $\mathcal{G}^{+++}$invariant formulation of gravity and $M$-theories: exact BPS solutions, J. High Energy Phys. 01 (2004) 002, hep-th/0311255.

[13] S. Elitzur, Impossibility of Sponteanously Breaking Local Symmetries, Phys. Rev. D12 (1975) 3978.

[14] T. Damour, M. Henneaux and H. Nicolai, $E_{10}$ and a small tension expansion of $M$-theory, Phys. Rev. Lett. 89 (2002) 221601, hep-th/0207267.

[15] P. West, Very Extended $E_{8}$ and $A_{8}$ at low levels, gravity and supergravity, Class. Quant. Grav. 20 (2003) 2393, hep-th/0212291.

[16] A. Kleinschmidt, I. Schnakenburg and P.West, Very-extended Kac-Moody algebras and their interpretation at low levels, hep-th/0309198.

[17] R. Argurio, F. Englert and L. Houart, Intersection rules for p-branes, Phys. Lett. B398 (1997) 61, hep-th/9701042.

[18] E. Witten, bound states of strings and p-branes, Nucl. Phys. B460 (1996) 335, hep-th/9510135.

[19] C. Bachas, Lectures on D-branes, hep-th/9806199.

[20] X. Bekaert and A. Gomberoff, Quantization of Chern-Simons coupling constant, J. High Energy Phys. 01 (2003) 054, hep-th/0212099.

[21] C. Pope, Lectures on Kaluza-Klein, http://faculty.physics.tamu.edu/ pope/.

[22] E. Cremmer, B. Julia, H. Lü and C. N. Pope, Higher dimensional origin of D=3 coset symmetries, hep-th/9909099.

[23] M. Gaberdiel, D. Olive and P. West, A class of Lorentzian Kac-Moody algebras, Nucl. Phys. B645 (2002) 403, hep-th/0205068.

[24] P. West, Hidden superconformal symmetries of M-theory, J. High Energy Phys. 08 (2003) 007, hep-th/0005270.

[25] V. Kac, Infinite dimensional Lie algebras, 3rd edition. Cambridge University Press (1995).

[26] P. West, $E_{11}, S L(32)$ and Central Charges, Phys. Lett. B575 (2003) 333, hep-th/0307098.

[27] A. Kleinschmidt and P. West, Representations of $\mathcal{G}^{+++}$and the role of space-time, J. High Energy Phys. 02 (2004) 033, hep-th/0312227. 